\title{
Spinal extradural arteriovenous malformations with parenchymal drainage: venous drainage variability and implications in clinical manifestations
}

\author{
Michelle J. Clarke, M.D., ${ }^{1}$ Todd A. Patrick, M.D. ${ }^{1}$ Ph.D., J. Bradley White, M.D., \\ Ph.D., ${ }^{1}$ Harky J. Cloft, M.D., Ph.D., ${ }^{2}$ William E. Krauss, M.D., ${ }^{1}$ E. P. Lindell, M.D., ${ }^{2}$ \\ and David G. Piepgras, M.D. ${ }^{1}$ \\ Departments of ${ }^{1}$ Neurologic Surgery and ${ }^{2}$ Radiology, Mayo Clinic, Rochester, Minnesota
}

\begin{abstract}
Object. Although nontraumatic spinal arteriovenous malformations and fistulas (AVMs and AVFs) restricted to the epidural space are rare, they can lead to significant neurological morbidity. Careful diagnostic imaging is essential to their detection and the delineation of the pathological anatomy. Aggressive endovascular and open operative treatment can provide arrest and reversal of neurological deficits.

Methods. The authors report on 6 cases of extradural AVMs/AVFs causing progressive myelopathy. Clinical findings, diagnostic evaluation, treatment, and outcome are discussed. Special consideration is given to the anatomy of the lesions and the operative techniques used to treat them. A review of the literature concerning extradural vascular malformations is also presented.

Results. All 6 cases of extradural AVMs had an extradural fistulous location with intradural medullary venous drainage. These cases illustrate progressive myelopathy through cord venous congestion (hypertension) that can be caused by an extradural nidus or fistula. In 4 cases, a large epidural lake was identified on angiography. At surgery, the epidural lake was obliterated and medullary drainage interrupted. All patients had stabilization of their neurological deficits and successful obliteration of the AVM/AVF was obtained.

Conclusions. Extradural AVMs and AVFs are a poorly described entity with published clinical experience limited to sporadic case reports and small series. Although these lesions have a purely extradural location of arteriovenous shunting and early venous drainage, they can be responsible for acute and progressive neurological symptoms similar to those caused by their dural-based intradural counterparts. With careful imaging recognition of the pathological anatomy, surgical and endovascular techniques can be used for the treatment of extradural AVMs affording effective and durable obliteration with stabilization or reversal of neurological symptoms. Venous drainage directly correlates the pathologic mechanisms of presentation. Specific attention must be paid intraoperatively to the epidural lake common to both variants so that recurrence is avoided. (DOI: 10.3171/FOC.2009.26.1.E5)
\end{abstract}

KEY WORDS $\bullet$ dural arteriovenous fistula $\bullet$
extradural arteriovenous malformation $\bullet$ intraspinal angioma
spinal arteriovenous fistula $\bullet \quad$ spinal arteriovenous malformation

$\mathrm{S}$ PINAL vascular malformations and fistulas are classified based on their vascular supply, vascular drainage, and nidus location. Although relatively uncommon lesions, they are clinically important because they can produce neurological symptoms caused by hemorrhage, ischemia, and mass effect. ${ }^{12}$ Selective spinal angiography has led to a better understanding of this pathological entity.

Arteriovenous shunting occurs with direct communication between the arterial and venous system. These can be plexiform AVMs (collections of dysplastic vessels

Abbreviations used in this paper: $\mathrm{AVF}=$ arteriovenous fistula; $\mathrm{AVM}=$ arteriovenous malformation; $\mathrm{dAVF}=$ dural $\mathrm{AVF}$. supplied by feeding arteries and drained by venous channels) or AVFs (feeding artery and draining vein without an intervening nidus). The many shunt types and lesions locations have led to a number of classification systems.

Oldfield and Doppman ${ }^{10}$ classified spinal vascular malformations into 4 types. Type 1 is a dAVF, usually arising near the nerve root sleeve. Typically, retrograde drainage occurs via the spinal medullary veins, resulting in intramedullary venous hypertension and engorged veins on the surface of the cord. Type 2 lesions are glomus AVMs made up of a distinct mass of dysmorphic arteries and veins without a capillary bed. These may be partially or entirely intramedullary. Type 3, juvenile AVMs, are congenital malformations consisting of arterial and venous networks without a distinct nidus. Often extensive, 
M. J. Clarke et al.
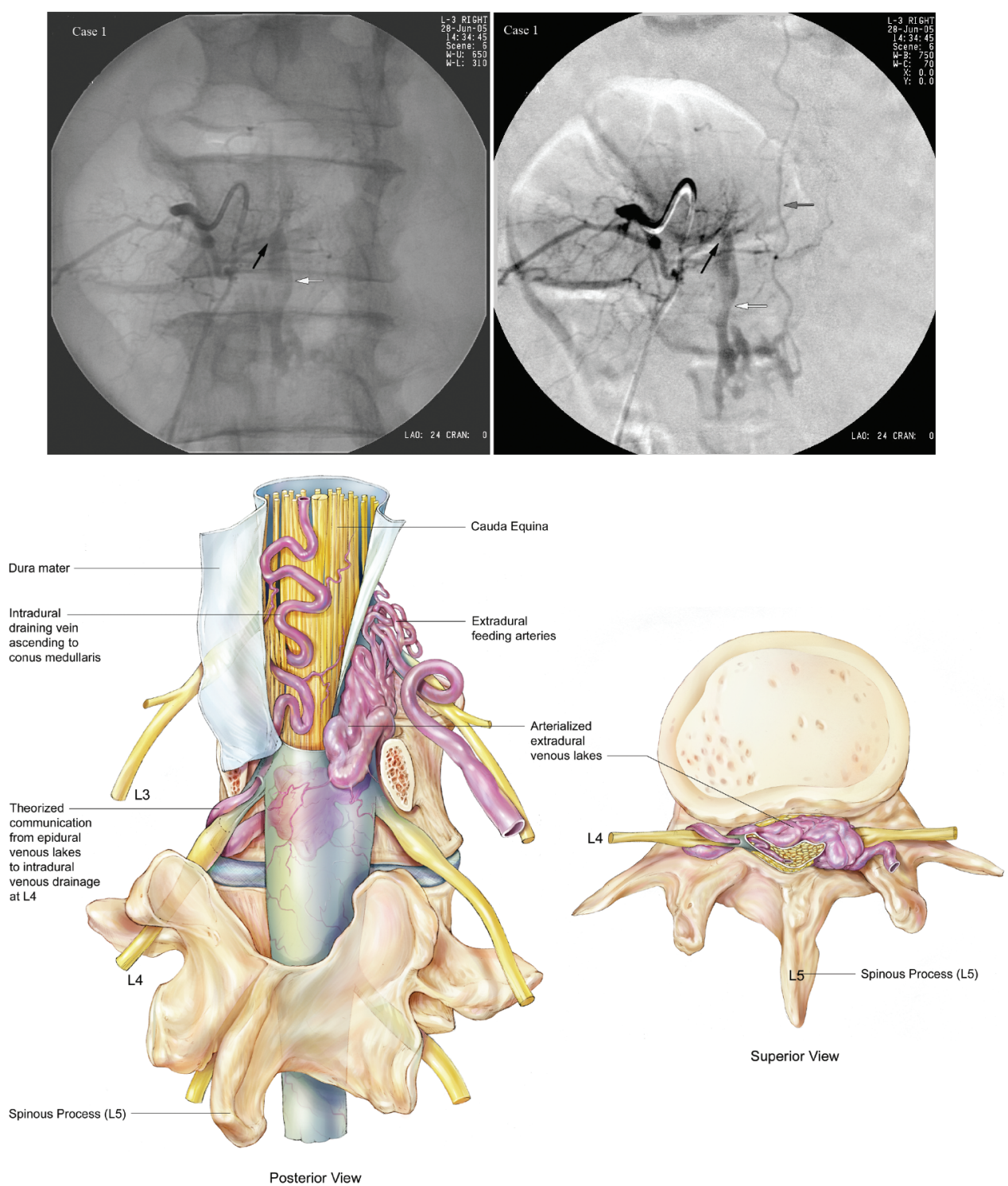

Fig. 1. Case 1. Early scout angiogram (A) and late angiogram (B) with right $L-3$ artery injection, depicting an extradural AVF (black arrow) with venous pouch (white arrow), with intradural drainage via the left L-4 nerve root sheath (gray arrow). Artist's impression of the same AVF is also shown (C).

these variably involve the spinal cord, and vertebral and paraspinal tissues (metameric AVMs). Type 4 AVMs, or perimedullary fistulas, are intradural AVFs located on the spinal cord surface with medullary venous drainage.

Spinal AVMs and AVFs confined to the epidural (also termed extradural) space and combined intraduralextradural lesions are rare and do not fall into the above classification system, yet they represent a neurologically relevant location of arteriovenous shunting that is anatomically distinct from typical spinal dAVFs. Spetzler and colleagues ${ }^{13}$ proposed a reclassification of spinal cord vascular lesions to include extradural variants and to designate such lesions as extradural rather than epidural to hopefully minimize confusion. These extradural lesions may cause myelopathy through a combination of the potential mechanisms including compression by ve- nous channels, venous congestion, "vascular steal," and conceivably hemorrhage. ${ }^{8}$

Current understanding of extradural AVMs is derived from case reports and small case series that elaborate on their potential to cause devastating epidural hemorrhages or progressive myelopathy due to venous hypertension or cord compression. Our experience leads us to believe that 2 distinct types of extradural AVMs exist: pure extradural AVMs as described by Spetzler et al.,13 and extradural AVMs and AVFs with retrograde medullary venous drainage with the fistula site located at some distance from the dural penetration site. Additionally, there are mixed- or juvenile-type true vascular malformations that involve both intradural and extradural compartments extensively. Our discussion is limited to extradural AVMs with medullary venous drainage. 


\section{Spinal extradural AVMs with parenchymal drainage}

\section{Illustrative Cases}

\section{Case 1}

This 65-year-old man presented with a 3-month history of subacute progressive lower extremity weakness and urinary retention. Initial T2-weighted MR images demonstrated signal changes and expansion of the thoracolumbar spinal cord and an abnormal intraspinal vascular structure. On the day he was transferred to our institution, the patient became acutely paraplegic. The patient underwent MR angiography that demonstrated an abnormal vascular structure near the inferior surface of the right L-3 pedicle, which allowed a focused spinal angiogram (Fig. 1A and B). Injection of the right L-3 lumbar artery filled an extradural AVF that fed into a venous pouch which drained via the anterior epidural venous plexus contralaterally to the left L-4 nerve sheath, where it coursed intradurally, ascending toward the medullary conus.

Anatomical findings consistent with those on preoperative angiography were found at surgery. Hypertrophied arteries adjacent to the right L-3 root fed a large venous channel in the right epidural space. This was incised with the release of pulsatile flow consistent with arterialization of the epidural venous complex. Exploration of the venous channel revealed no communicating vessel penetrating the common dural sac in the right lateral recess. A midline durotomy was performed from L-2 to L-4, and a number of enlarged, congested veins were seen in the cauda equina. A prominent vascular channel ascending within the cauda equina was identified and proved to be an arterialized vein entering the dura mater with the left L-4 nerve root (Fig. 1C). This arterialized vein was coagulated and divided intradurally at the L-4 entry site. All extradural arterial channels in the extradural venous plexus around the right L-3 nerve root were then coagulated and disconnected, and the extradural arterialized varix was resected. Intradural inspection thereafter demonstrated the congested veins to be darker, consistent with obliteration of fistula and retrograde flow. The patient did well postoperatively, regaining some strength and mobility. He lives independently but remains wheelchair-bound.

\section{Case 2}

This 70-year-old man presented with a 5-month history of progressive leg weakness, decreased perineal sensation, and urinary retention. An MR image showed T2 signal changes and marked enlargement of the lower thoracic spinal cord. Angiography demonstrated what appears to be a dAVF arising from the left L-4 lumbar artery, with prominent extramedullary ascending venous drainage. Operative exploration was performed, but intradural exposure of the L-4 and L-5 levels failed to reveal a draining vein.

Repeated angiography demonstrated that the L-4 lumbar artery supplied the extradural AVF. Also noted was an enlarged L-4 dural branch supplying a serpiginous vein in the anterior epidural venous plexus, which ultimately drained intradurally via radicular veins at the L2-3 level. The AVF was obliterated endovascularly with
$\mathrm{N}$-butyl 2-cyanoacrylate polymer during the same session. Follow-up images obtained 23 months later confirmed obliteration, and T2-weighted MR images demonstrated resolution of the signal changes and mass effect in the spinal cord. At the 2-year follow-up examination, the patient was significantly improved, with only mild, residual proximal leg weakness and neurogenic bladder.

\section{Case 3}

This 61-year-old woman presented with a 7-month history of progressive burning calf pain and perianal numbness. The patient was initially treated with steroids and rehabilitation therapy with slight improvement, but further weakness ensued 3 months prior to presentation. At presentation she had profound weakness in both lower extremities, neurogenic bowel and bladder dysfunction, and an L-2 sensory level bilaterally, including absent sacral sensation.

Magnetic resonance images demonstrated conus edema and dilated vessels on the dorsal surface of the cord consistent with an AVF. Angiography results were originally described as a dAVF supplied via the right L-3 radiculomedullary artery. Retrograde flow through a medullary vein ascended to the conus, resulting in dilation of the pial plexus. A $4 \times 10-\mathrm{mm}$ collection of contrast enhancement was noted arising from the L-3 lumbar artery thought to represent a small aneurysm.

Initially after hemilaminectomy, there was no evidence of any vascular lesion surrounding the right L-3 root, or in the anterior epidural space. The dura was opened, and an arterialized vein entering with the right L-3 root, which ascended to the cauda equina, was coagulated and cut. The epidural space was again explored in an attempt to locate the suspected venous aneurysm; however, only an enlarged epidural plexus was noted. The epidural plexus was opened, and the pulsatile pressure noted was consistent with the presence of an extradural AVF. Attempts at recording vascular pressure in the venous plexus were unsuccessful. The sac was obliterated with packing and confirmed on follow-up angiography.

Magnetic resonance images obtained 22 months postoperatively demonstrated resolution of the previously noted edema in the conus and thoracic spinal cord. The serpiginous vessels had also resolved. At the 4-year follow-up examination, the patient was ambulatory with a cane, having recovered some of the sensory and motor function, and having regained bladder sensation.

Case 4

This 77-year-old man, who had undergone decompressive lumbar laminectomy 30 years earlier, presented 5 months after sudden onset of ascending lower extremity paresthesias, weakness, and bladder and bowel dysfunction. The results of evaluation and workup at another institution, which included CT myelography and MR imaging, suggested a possible dAVF. The CT myelogram demonstrated subtle serpentine filling defects along the ventral and dorsal aspect of the spinal cord from T-6 to L-1 that were consistent with a dilated posterior pial plexus draining a dAVF. Magnetic resonance imaging re- 
sults were unremarkable. On transfer to our institution, an angiogram demonstrated an apparent dAVF at left L-3, originating from branches of the lumbar artery. Furthermore, it appeared that this fistula extended to involve the epidural venous plexus with venous drainage following the left L-3 radicular vein to ascend in the anterior medullary vein to the level of T-9.

The patient underwent a multilevel lumbar laminectomy. Numerous dilated vessels were seen surrounding the left L-2 and L-3 roots. The veins above and below the root, and the dilated epidural veins were coagulated. One epidural vein was incised, releasing high-pressure arterialized blood. This vein was cannulated with a fine Teflon needle, and an attached transducer demonstrated pressures equal to the systemic arterial pressure. The veins were cauterized and packed. The dura mater was opened, and an arterialized vein traveling with the left L-3 root was clipped and divided. The patient did well, and was ambulatory with normal lower extremity sensation and bladder function at last follow-up 10 years after surgery.

\section{Case 5}

This 53-year-old woman presented with progressive weakness and sensory loss in the lower extremities, and an episode of bladder incontinence 4 weeks prior to admission. An MR image demonstrated a T2 signal abnormality extending from the upper thoracic cord to the medullary conus. Magnetic resonance angiography confirmed the presence of venous tortuosity through the full length of the visualized cord, including an abnormal vascular complex at the left L5-S1 foramen (Fig. 2A and B). This immediately abutted an area of contrast loculation in the left ventral epidural space extending to the S-1 foramen, appeared to enter the dura and continued as the radicullomedulary vein, which continued cephalad. Subsequent angiography substantiated these findings. The left L-4 lumbar artery contributed to the fistula, located at the expected L-5 root sleeve. Drainage occurred in the epidural space inferiorly to $\mathrm{S}-1$ and then ascended to enter the dura at the L-5 level (Fig. 2C).

The patient underwent L4-S1 laminectomies, and abnormal vessels in the axilla of the left L-5 root were identified. These vessels crossed between the common dural sac and L-5 nerve root, and between the fistulous abnormality anterior to the common dural sac and L-5 nerve root. Caudally, an arterialized epidural varix was associated with extensive bone erosion. The abnormal vessels entering the site of the fistula and the veins exiting the varix were coagulated and the fistula was resected. Finally, the large venous complex arising from the inferior varix, which was blue by this time, was coagulated and divided. The dural tube was not opened. Immediate postoperative angiography demonstrated complete obliteration of the fistula (Fig. 2D).

FIG. 2. Case 5. A and B: Magnetic resonance angiography showing epidural pouch (arrows). C: Angiogram demonstrating L-4 injection draining inferiorly to $S-1$ in the epidural space before entering the dura at L-5. The fistula site is marked with a black arrow, the epidural lake with a gray arrow, and the draining vein with a white arrow. D: Nondiagnostic angiogram obtained after the first surgery. E: Follow-up angiogram depicting a new AVF.
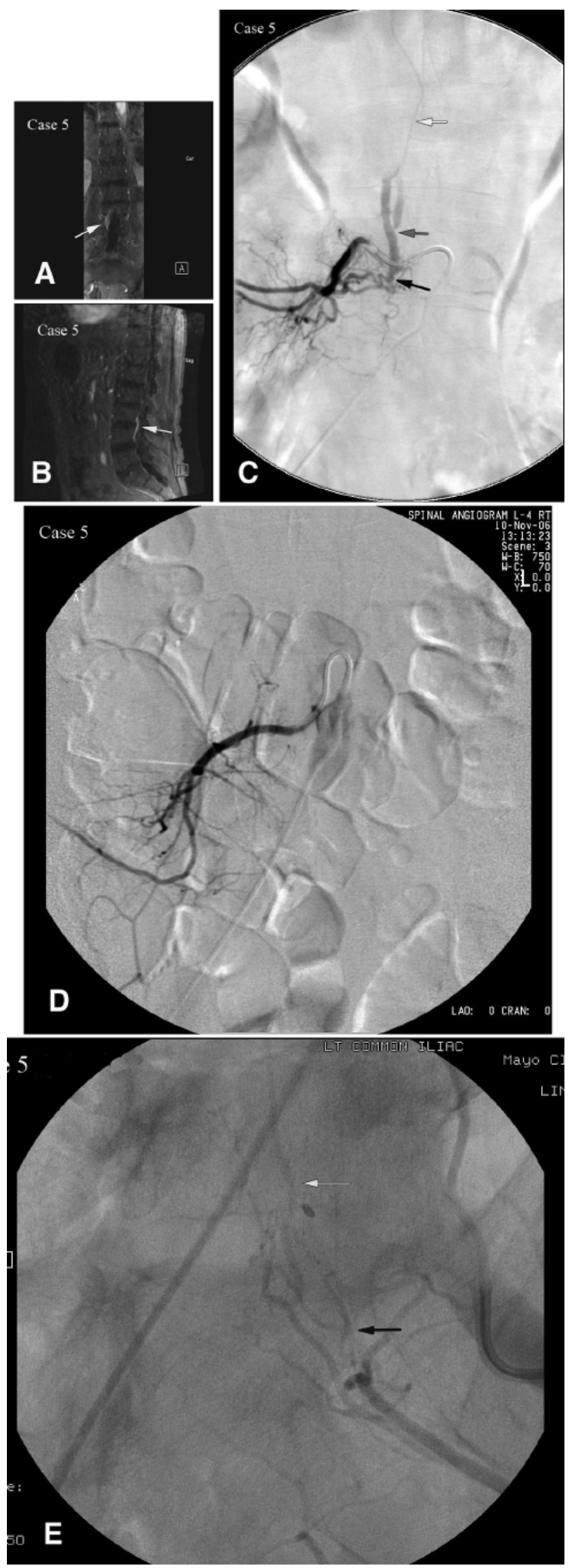


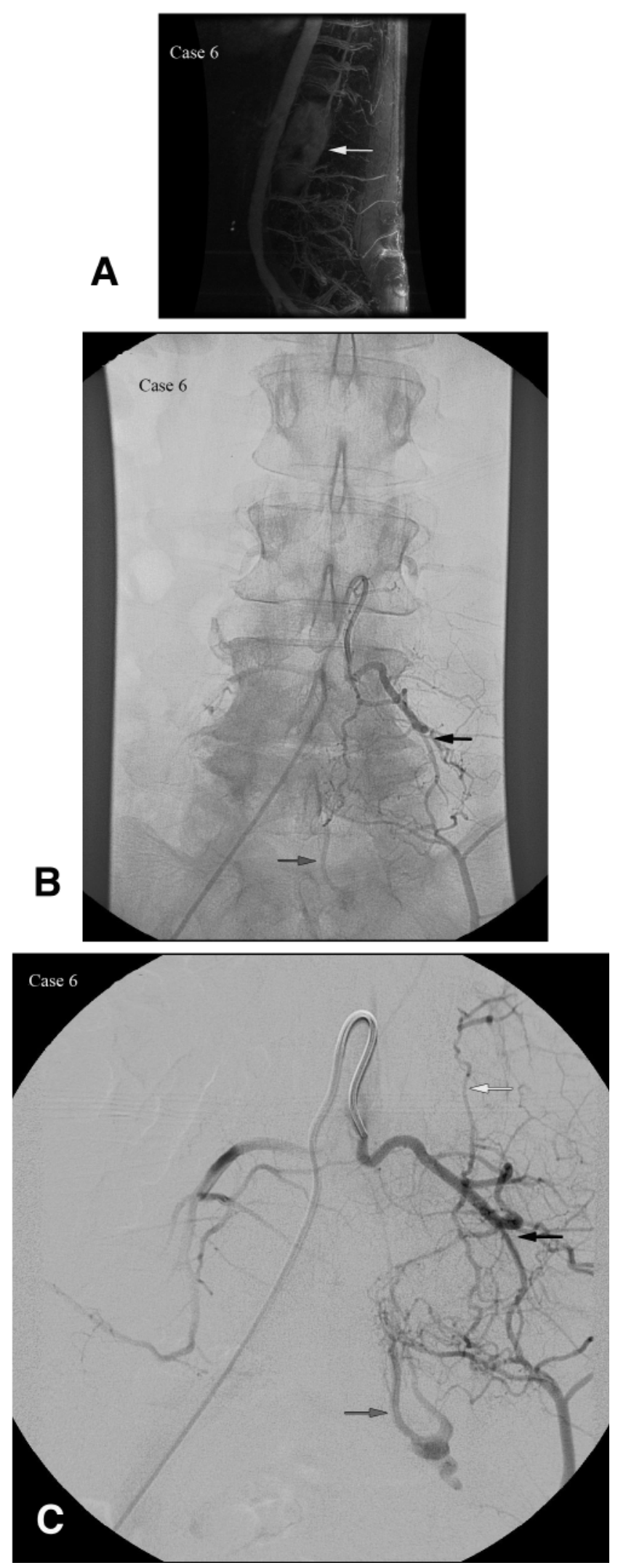

FIG. 3. Case 6. A: Magnetic resonance angiography scout image showing epidural lake (arrow). B and C: Angiograms demonstrating L-4 AVF (black arrow) with large epidural lake (gray arrow) and intradural drainage at the L-3 level (white arrow).

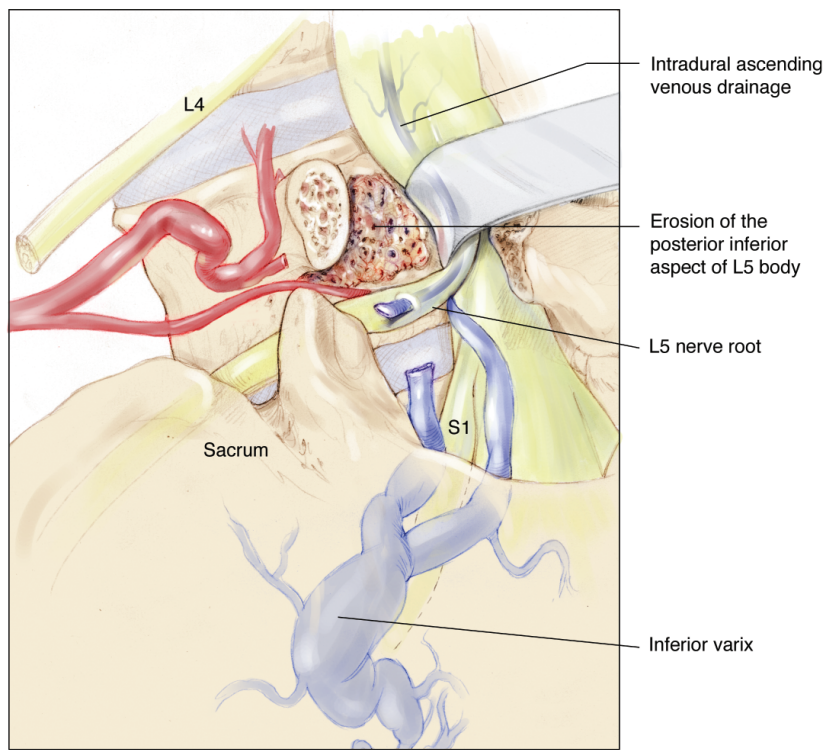

FIG. 4. Case 6. An artist's impression of the AVF.

The patient noted immediate improvement in strength, sensation, and bowel and bladder function. Unfortunately at 3 months, the results of postoperative MR angiography suggested possible recanalization with a persistent arterialized radicular medullary vein entering from the left L5-S1 foramen, with multiple enlarged veins and signal changes visible on T2-weighted images of the thoracolumbar cord (Fig. 2E).

The patient's previous laminectomy scar was reopened and the L-5 and S-1 roots were identified; however, extensive scarring precluded exploration of the epidural component of the AVF. A durotomy allowed identification of a large arterialized vein traveling with the S-1 rootlets, which matched the identified ascending draining vein on the previous angiogram. This vein was coagulated and divided. Repeated angiography demonstrated obliteration of the AVF. The patient continued to do well and was discharged home. Since her nondiagnostic postoperative angiogram, the patient has undergone routine follow-up with MR angiography without evidence of recurrence.

\section{Case 6}

This 76-year-old man presented with a 7-month history of gradually progressive bilateral lower extremity weakness, paresthesias, and dysesthesias. Three days prior to admission, the patient developed complete lower extremity function loss and bowel and bladder dysfunction. Magnetic resonance imaging and angiography revealed nonspecific signal abnormalities on $\mathrm{T} 2$-weighted images of the midcervical cord to the medullary conus, and prominent vascularity including serpentine dorsal medullary veins (Fig. 3A). Spinal angiography demonstrated a fistula under the right L-4 pedicle. This fistula connected to a large draining vein in the right ventral epidural plexus, which transitioned intradurally at L-3 to a smaller vein with a more typical appearance in the dorsal surface of the spinal cord and extending up to the lower cervical spine (Fig. 3B and C; Figs. 4 and 5). 


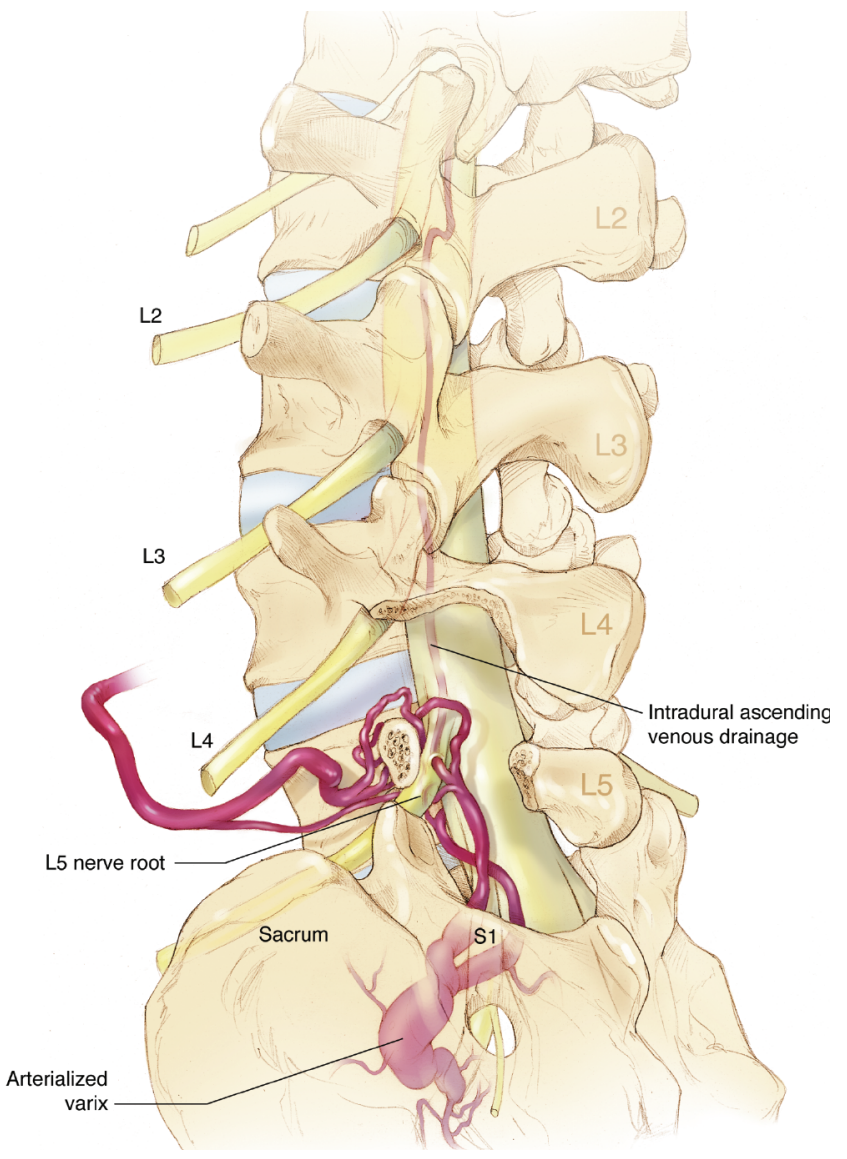

FIG. 5. Case 6. An artist's impression of the AVF.

The patient underwent laminectomy and durotomy at the predicted lesion site. Two obviously abnormal arterialized veins were noted to penetrate the dura near the right L-3 root. The fistula was cauterized and sectioned. The extradural spaces to the right of L-3 and L-4 were explored, and abnormal epidural vessels were also cauterized and sectioned. Arteriovenous fistula obliteration was confirmed on follow-up angiography.

\section{Discussion}

Arteriovenous malformations and fistulas of the epidural space with retrograde medullary venous drainage are rare. Blood normally flows from the segmental arteries to the spinal medullary arteries supplying the spinal arteries and radicular arteries supplying the nerve root dural sleeve. Venous drainage occurs from the subarachnoid space to the valveless epidural venous plexus. In a typical dAVF, the fistulous vessel penetrates the dura at the fistula site. Unlike most dAVFs, however, in those we termed extradural, the fistulous vessels did not directly penetrate the dura at that immediate site, but possessed an extradural component. It is possible that in some fistulas the epidural venous system offers an alternative means of arterialized venous egress, and that these alternative systems are only detected when there is a mass effect or retrograde flow causing cord edema.

It is important to recognize these unique malforma- tions preoperatively to ensure complete treatment. Although similar entities have been described in the literature, mistaking such a lesion as purely epidural may result in insufficient treatment and recurrence. It is imperative that the feeding and draining vessels are clearly defined preoperatively, and that both the intra- and extradural components of the fistula are surgically addressed.

Nontraumatic AVMs and AVFs involving the extradural space are uncommon lesions known only through case reports. ${ }^{5,13}$ It is possible that these extradural AVM/ AVFs arise as congenital or acquired lesions; interestingly, the patient in Case 4 developed a fistula 30 years following laminectomy, supporting the possibility that these lesions are acquired. Purely extradural AVFs have been associated with penetrating and nonpenetrating trauma, neurofibromatosis, ${ }^{7}$ and other more extensive vascular malformations.

There are 2 main causes of neurological deficits with this lesion: mass effect and medullary venous hypertension. $^{2-4,11,13}$ Neurological deficits may occur as a result of mass effect exerted on the spinal cord by the expanding extradural varix. This mechanism can occur in extradural AVM/AVFs with or without intramedullary drainage. However, all lesions in this series had an intradural component. A completely extradural fistula of the epidural venous plexus with retrograde venous drainage into the intradural compartment occurs, causing venous congestion and medullary venous hypertension. Other mechanisms such as intraspinal hemorrhage ${ }^{9}$ and medullary blood flow steal leading to ischemia have been theorized, but not proven. ${ }^{8}$ Additionally, the necrotic myelopathy FoixAlanjouanine Syndrome may occur.

In all of the cases we have described, the patients suffered a moderate-to-severe deficit with a subacute to rapid deterioration. In many cases, a diagnostic delay occurred, which also delayed treatment and allowed the patients' conditions to deteriorate further. Thus, it is important to review the syndrome of medullary venous hypertension seen in most patients with spinal AVFs, including telltale asymmetry and mixed upper and lower motor neuron signs. Patients often experience distal-to-proximal paresthesias and dysesthesias, progressive weakness ranging from giveway to paraparesis, and sphincter dysfunction (predominantly bladder).

As demonstrated by the 6 cases described, recognition of this lesion is aided by MR imaging, which often demonstrates T2 signal changes attributable to edema resulting from venous congestion. Perimedullary flow voids also aid in diagnosis but must be distinguished from cerebrospinal fluid-related flow artifact, and MR angiography may demonstrate abnormal intra- and extradural vascularity. Selective arterial angiography remains the gold standard for determining the exact nature of the fistula. Because these lesions are rare and difficult to find, surgeons and neuroradiologists must maintain a high index of suspicion for their existence. In addition, it is important to note there is wide anatomic variety in these lesions: there may be multiple feeding vessels, fistula sites, and venous drainage patterns.

To provide long-term symptom control, the lesions must be completely obliterated and/or excised; ${ }^{1}$ partial 
treatment carries the risk that the extradural AVM will recruit a new blood supply and eventually reexpand to become symptomatic. ${ }^{6}$ In their retrospective series, Afshar and colleagues ${ }^{1}$ treated 2 patients with both intradural and extradural venous drainage by surgically interrupting the intradural draining vein, but with demonstrated persistent flow in the extradural veins. In 1 case, the extradural AVF spontaneously thrombosed, however, intrathecal drainage was reestablished in the other patient. In addition, in our series, the patient in Case 6 had a recurrence after obliteration of only the extradural component of the AVM.

In the remaining cases presented, both intra- and extradural drainage was eliminated, either with surgery or through embolization. Durability appears good as clinical or radiographic recurrences have not developed in any of our patients. Although embolization may play a role in the obliteration of these lesions, particularly in purely extradural AVMs, operative obliteration will probably remain the most reliable, definitive therapy. Although embolization ${ }^{14,15}$ or operation alone has been used in treatment of these lesions, combined therapy may offer the best prospect for long-term resolution in more complex cases.

\section{Conclusions}

Currently, extradural vascular malformations are excluded in the common classification system of spinal vascular malformations. These case reports emphasize that although rare, these lesions have the potential to cause serious neurological symptoms. We believe that treatment should be directed toward complete obliteration of intradural lesions.

\section{Disclaimer}

The authors report no conflict of interest concerning the materials or methods used in this study or the findings specified in this paper.

\section{Acknowledgment}

The authors thank Dr. David F. Kallmes, Division of Neurointerventional Radiology at the Mayo Clinic, for his assistance.

\section{References}

1. Afshar JKB, Doppman JL, Oldfield EH: Surgical interruption of intradural draining veins as curative treatment of spinal dural arteriovenous fistulas. J Neurosurg 82:196-200, 1995
2. Arnaud O, Bille F, Pouget J, Serratrice G, Salamon G: Epidural arteriovenous fistula with perimedullary venous drainage: case report. Neuroradiology 36:490-491, 1994

3. Chen CJ, Huang CC, Hsu YY, Hsu WC: Small, isolated paraspinal arteriovenous fistula. AJNR Am J Neuroradiol 18:359-361, 1997

4. Cognard C, Semaan H, Bakchine S, Miaux Y, Sola-Martinez MT, Chiras J: Paraspinal arteriovenous fistula with perimedullary venous drainage. AJNR Am J Neuroradiol 16:20442048, 1995

5. Goyal M, Willinsky R, Montanera W, terBrugge K: Paravertebral arteriovenous malformations with epidural drainage: clinical spectrum, imaging features, and results of treatment. AJNR Am J Neuroradiol 20:749-755, 1999

6. Hemphill JC, Smith WS, Halbach VV: Neurologic manifestations of spinal epidural arteriovenous malformation. Neurology 50:817-819, 1998

7. Hoffman HB, Bagan M: Cervical epidural arteriovenous malformation occurring with a spinal neurofibroma. J Neurosurg 26:346-351, 1967

8. Miyagi Y, Miyazono M, Kamikaseda K: Spinal epidural vascular malformation presenting in association with a spontaneously resolved acute epidural hematoma. Case report. J Neurosurg 88:909-911, 1998

9. Muhonen MG, Piper JG, Moore SA, Menezes AH: Cervical epidural hematoma secondary to an extradural vascular malformation in an infant: case report. Neurosurgery 36:585587,1995

10. Oldfield EH, Doppman JL: Spinal arteriovenous malformations. Clin Neurosurg 34:161-183, 1988

11. Pirouzmand F, Wallave MC, Willinsky R: Spinal epidural arteriovenous fistula with intramedullary reflux. J Neurosurg 87:633-635, 1997

12. Sharma RR, Selmi F, Cast IP, O’Brien C: Spinal extradural arteriovenous malformation presenting with recurrent hemorrhage and intermittent paraplegia: case report and review of the literature. Surg Neurol 42:26-31, 1994

13. Spetzler RF, Detwiler PW, Rhna HA, Porter RW: Modified classification of spinal cord vascular lesions. J Neurosurg (2 Suppl) 96:145-156, 2002

14. Szajner M, Weill A, Piotin M, Moret J: Endovascular treatment of a cervical paraspinal arteriovenous malformation via arterial and venous approaches. AJNR Am J Neuroradiol 20:1097-1099, 1999

15. Willinsky R, terBrugge K, Montanera W, Wallace MC, Gentili F: Spinal epidural arteriovenous fistulas: arterial and venous approaches to embolization. AJNR Am J Neuroradiol 14:812-817, 1993

Manuscript submitted July 20, 2008.

Accepted October 13, 2008.

Address correspondence to: Michelle J. Clarke, M.D., Department of Neurologic Surgery, Mayo Clinic, Rochester 200 First Street SW, Rochester, Minnesota 55905. email: Clarke.michelle@mayo.edu. 